\title{
Lung transplant recipients receiving voriconazole and skin squamous cell carcinoma risk in Australia
}

\begin{abstract}
Chin Fen Neoh BPharm, MPharm(ClinPharm), Senior Lecturer

Gregory I Snell MBBS, FRACP, MD
Medical Head, Lung Transplant Service 2

Bronwyn Levvey RN, BEdStu, GradDipClinEpi Team Leader, Lung Transplant ${ }^{2}$

Tom Kotsimbos MD, FRACP Respiratory and Transplant Physician ${ }^{2}$

Orla Morrissey MBBCh. FRACP PhD Infectious Diseases Physician ${ }^{3}$

Monica A Slavin

MBBS, FRACP, MD Head, Infectious Diseases ${ }^{4}$

\section{Kay Stewart BPharm, PhD Adjunct Associate Professor ${ }^{5}$}

Clinical record

In April 2002, 3 months after her second bilateral lung transplantation, a 45-year-old female patient commenced treatment for necrotising Aspergillus tracheobronchitis with liposomal amphotericin B followed by voriconazole $200 \mathrm{mg}$ twice daily for 13.2 months. In January 2003, mild erythema was noted on her forehead and cheeks, accompanied by dryness and scaling of her forearms and dorsa of her hands. A photosensitive drug reaction was suspected. Two months later, several skin squamous cell carcinoma (SCC) lesions and solar keratoses were noted on the dorsa of her hands. In May 2003, voriconazole administration was ceased because the fungal infection had resolved.

In November 2004, the patient commenced a second course of voriconazole $200 \mathrm{mg}$ twice daily - again, to treat tracheobronchitis - which was continued for 10.6 months. Three months after she started treatment, a photosensitive rash was noted on her lower legs and forearms. In September 2005, six skin SCC lesions were excised from her forehead and left hand, and actinic keratosis lesions were noted on her right forehead, the backs of her hands and on her chest and ankles. In January 2006, a third course of voriconazole $200 \mathrm{mg}$ twice daily was administered for 7.2 months to manage Aspergillus airways colonisation. Two months after initiation of voriconazole prophylaxis, three small unspecified skin lesions were observed on her right temple, nose and anterior chest.
\end{abstract}

David CM Kong BPharm, MPharm, PhD Lecturer $^{5}$

1 Clinical Pharmacy, Universiti Teknologi MARA, Puncak Alam, Selangor, Malaysia.

2 Allergy, Immunology and

Respiratory Medicine,

Alfred Hospital and Monash University, Melbourne, VIC.

$\mathbf{3}$ Infectious Diseases, Alfred Health and

Monash University, Melbourne, VIC.

4 Infectious Diseases and Infection Control, Peter MacCallum Cancer Centre, Melbourne, VIC.

5 Centre for Medicine Use and Safety, Monash University, Melbourne, VIC.

david.kong@ monash.edu
The patient underwent a third bilateral lung transplantation in September 2006 and received posaconazole prophylaxis for nearly 2 years. Multiple actinic keratosis lesions were again observed on her forehead, chest and hands. Twelve months later, she was diagnosed with a parotid gland metastasis from an SCC. She also developed subcutaneous nodules on the forehead, consistent with in-transit metastatic SCC. Radiotherapy was initiated to treat locally invasive skin SCC. In January 2008, a dermal lesion just to the left of the central forehead scar appeared as a new dermal metastasis. Skin SCC lesions were also noted on both of her lips, her left index and right little finger, and there were multiple actinic keratosis lesions on her hands, chest and neck.

In April 2008, the patient underwent right parotidectomy. The histopathology report showed residual microscopic skin SCC deep in the intratemporal fossa region, indicating a high risk of tumour recurrence. Oral capecitabine was given in conjunction with radiotherapy. In October 2008 ( 2 months after treatment with posaconazole was ceased), she again developed several actinic keratosis lesions scattered on her hand and forehead. She started a second course of posaconazole in December 2008. The patient experienced ongoing facial pain with recurrent tumour in and around the trigeminal nerve. She received stereotactic radiotherapy for meningioma involving the right middle cranial fossa in March 2009. The patient died, owing to metastatic SCC, in October 2009 merging evidence for causal associations between prolonged voriconazole exposure and skin SCC ${ }^{1-6}$ is of concern, given the frequent use of voriconazole prophylaxis, administered for months in patients after lung transplantation (LTx). ${ }^{7}$ In our institution, $13.7 \%$ of patients (14/102) receiving voriconazole after LTx between July 2003 and June 2010 had at least one episode of skin SCC. Drug-related photosensitivity is the most common cutaneous reaction that has been reported with voriconazole use. ${ }^{8}$ It has been postulated that long-term voriconazole therapy results in chronic phototoxicity, which, in turn, contributes to the development of skin SCC in transplant recipients. ${ }^{1}$

A study in the United States from 2003-2008 3 reported that a high cumulative voriconazole dose was not an independent risk factor for skin SCC in patients who have undergone LTx, in contrast to other findings. ${ }^{4}$ However, this same study reported that the occurrence of skin SCC among LTx patients was related to the duration of voriconazole therapy, ${ }^{3}$ which is supported by more recent findings. 5,6 The inconsistencies between studies are likely to be related to differences in the methods employed to evaluate predictors and in study design, which may not have adequately controlled for potential confounding factors, such as patient sex, age, sun exposure, history of chronic obstructive pulmonary disease (which could be a proxy variable for smoking status) and level of immunosuppression. Longer and more intensive immunosuppressive regimens have been associated with a higher risk of developing skin SCC. ${ }^{9}$ Indeed, prolonged duration of voriconazole prophylaxis may have been a surrogate marker for a more compromised immune system or opportunistic infections that could, in turn, influence a patient's risk of developing skin SCC.

Residing in geographical locations with high levels of sun exposure has also been identified as an independent risk factor for LTx patients developing skin SCC. ${ }^{3}$ Higher rates of skin SCC have been reported in areas of significant sun exposure, with increased exposure to ultraviolet (UV) radiation being a significant risk factor. ${ }^{3}$ Importantly, UV radiation is a known distinct mutagen of keratinocytes and induces an immunosuppressed condition that prevents tumour rejection. ${ }^{10}$ In our patient, multiple skin SCC lesions were noted on photoexposed areas, as has been reported in other patients given prolonged voriconazole therapy. ${ }^{1,2}$ Our patient reported a history of extensive sun exposure and sunburn and was aged 45 years when her first skin SCC was diagnosed. This is consistent with evidence of a higher incidence of skin SCC in older populations, ${ }^{5}$ which could be explained by accumulation of high-dose UV radiation over a prolonged period of time.

It is worth noting that aggressive skin SCC lesions and the parotid gland metastasis in this patient occurred during posaconazole prophylaxis (after discontinuation of voriconazole). While studies have reported regression or fading of skin SCC after switching from voriconazole to posaconazole or itraconazole, ${ }^{2}$ this was not observed 


\section{Lessons from practice}

- Before commencing voriconazole therapy, obtain the patient's history of sun exposure and conduct a baseline skin review, with particular vigilance for patients with light skin, patients who have a high level of sun exposure and those with a previous history of skin SCC.

- Give the patient appropriate advice regarding sun protection (eg, wear sunscreen, hats and protective clothing).

- Monitor the patient for a photosensitivity reaction while they are receiving voriconazole therapy. If a reaction is noted, consider switching to an appropriate alternative.

- After voriconazole has been discontinued, conduct dermatological examinations at 3- to 6-month intervals, particularly for patients with previous skin SCC.

- The overall risks versus benefits of using voriconazole should be considered in patients with previous skin SCC and those who had a recurrence or worsening SCC lesions subsequent to voriconazole administration.

in our patient. Her skin SCC could have continued to develop spontaneously despite cessation of voriconazole or could have been due to prolonged posaconazole exposure. Clearly, we need to institute surveillance programs to ascertain whether posaconazole confers a similar level of risk to voriconazole with respect to the development of skin SCC.

In summary, long-term administration of voriconazole demands caution, especially in patients with a risk of high-level sun exposure. Routine prospective dermatological examination should be performed, particularly in patients at high risk as defined by the intensity and duration of immunosuppressive therapy, history of prior skin SCC and geographical location.
Acknowledgements: We thank staff from Health Information Services at the Alfred Hospital for their assistance with this study. Chin Fen Neoh gratefully acknowledges a PhD scholarship granted by the Universiti Teknologi MARA.

Competing interests: Orla Morrissey has sat on advisory boards for, received investigator-initiated grant support from and given lectures for Gilead Sciences, Pfizer, Merck, Schering-Plough and Orphan Australia. Monica Slavin has sat on advisory boards for and received research funding from Pfizer, Merck, Schering-Plough and Gilead Sciences. David Kong has sat on advisory boards for Pfizer and receives financial support from Pfizer, Novartis, Merck and Gilead Sciences.

1 Cowen EW, Nguyen JC, Miller DD, et al. Chronic phototoxicity and aggressive squamous cell carcinoma of the skin in children and adults during treatment with voriconazole. J Am Acad Dermatol 2010; 62: 31-37.

2 Epaulard O, Saint-Raymond C, Villier C, et al. Multiple aggressive squamous cell carcinomas associated with prolonged voriconazole therapy in four immunocompromised patients. Clin Microbiol Infect 2010; 16: 1362-1364.

3 Vadnerkar A, Nguyen MH, Mitsani D, et al. Voriconazole exposure and geographic location are independent risk factors for squamous cell carcinoma of the skin among lung transplant recipients. J Heart Lung Transplant 2010; 29: 1240-1244.

4 Singer JP, Boker A, Metchnikoff C, et al. High cumulative dose exposure to voriconazole is associated with cutaneous squamous cell carcinoma in lung transplant recipients. J Heart Lung Transplant 2012; 31: 694-699.

5 Feist $A$, Lee $R$, Osborne $\mathrm{S}$, et al. Increased incidence of cutaneous squamous cell carcinoma in lung transplant recipients taking long-term voriconazole. J Heart Lung Transplant 2012; 31: 1177-1181.

6 Zwald FO, Spratt M, Lemos BD, et al. Duration of voriconazole exposure: an independent risk factor for skin cancer after lung transplantation. Dermatol Surg 2012; 38: 1369-1374.

7 Neoh CF, Snell GI, Kotsimbos T, et al. Antifungal prophylaxis in lung transplantation - a world-wide survey. Am J Transplant 2011; 11: 361-366.

8 Patel AR, Turner ML, Baird K, et al. Voriconazole-induced phototoxicity masquerading as chronic graft-versus-host disease of the skin in allogeneic hematopoietic cell transplant recipients. Biol Blood Marrow Transplant 2009; 15: 370-376.

9 Jensen AO, Svaerke C, Farkas D, et al. Skin cancer risk among solid organ recipients: a nationwide cohort study in Denmark. Acta Derm Venereol 2010; 90: 474-479.

10 Grossman D, Leffell DJ. The molecular basis of nonmelanoma skin cancer: new understanding. Arch Dermatol 1997; 133: 1263-1270. 